\title{
First-line immunotherapy for patients with advanced stage or metastatic non-small cell lung cancer...finally what threshold of PD-L1 expression on tumor cells?
}

\author{
Paul Hofman ${ }^{1,2,3}$ \\ ${ }^{1}$ Université Côte d'Azur, CHU Nice, FHU OncoAge, Laboratory of Clinical and Experimental Pathology, Pasteur Hospital, Nice, France; \\ ${ }^{2}$ Université Côte d'Azur, CNRS, INSERM, IRCAN, FHU OncoAge, Nice, France; ${ }^{3}$ Université Côte d'Azur, CHU Nice, FHU OncoAge, Hospital- \\ Integrated Biobank (BB-0033-00025), Nice, France \\ Correspondence to: Paul Hofman, MD, PhD, Laboratory of Clinical and Experimental Pathology, Pasteur Hospital, 30 voie Romaine, BP69, 06001 \\ Nice cedex 01, France. Email: hofman.p@chu-nice.fr. \\ Comment on: Reck M, Rodríguez-Abreu D, Robinson AG, et al. Updated Analysis of KEYNOTE-024: Pembrolizumab Versus Platinum-Based \\ Chemotherapy for Advanced Non-Small-Cell Lung Cancer With PD-L1 Tumor Proportion Score of 50\% or Greater. J Clin Oncol 2019;37:537-46.
}

Submitted Apr 24, 2019. Accepted for publication Apr 28, 2019.

doi: $10.21037 /$ tlcr.2019.04.18

View this article at: http://dx.doi.org/10.21037/tlcr.2019.04.18

A certain number of patients with advanced stage or metastatic non-small cell lung cancer (NSCLC) show better overall survival when treated with first-line immunotherapy than patients treated with chemotherapy (1). This finding, originally reported in KEYNOTE-024, has just been updated and continues to confirm, for the same cohort of patients, benefit from first-line pembrolizumab treatment $(1,2)$. Immunohistochemical (IHC) expression of PD-L1 at a threshold of $50 \%$ positive tumor cells was used as a predictive biomarker to select patients $(1,2)$. The updated study by Reck et al., reports an average of overall survival of 30 vs. 14.2 months for patients treated with immunotherapy and chemotherapy, respectively (2). In the conclusion of this updated study it was reported that no cumulative toxicity was noted for prolonged treatment with pembrolizumab (2). Moreover, the number of fatal side effects induced by prolonged immunotherapy was low, only one patient developed irreversible lung disease leading to death (2).

A number of comments concerning the most recent study by Reck et al. should be raised. The results were obtained for patients selected based on parameters that may not correspond to those of the daily practice of hospitals and clinics $(2,3)$. In fact, the expression of PD-L1 was evaluated with tissue samples that excluded bronchial biopsies and cytological samples taken during bronchial endoscopy, particularly those obtained by guided echo-endoscopy (2). So, the IHC expression was analyzed on transthoracic biopsies, excised biopsy and surgical resected large specimens (2). The recruitment of patients was certainly biased with respect to the samples obtained $(2,3)$. The number of patients excluded due to insufficient material was not indicated in this publication, and the number and/or analyses of available transthoracic biopsies was not provided (2). The pharmDx 22C3 anti-PD-L1clone (Agilent, Santa Clara, CA, USA) was used. This was required, together with the use of a companion diagnostic test, by the guideline of the USA Food and Drug Administration for clinical trials. Even if standardized studies using different PD-L1 clones (in particular the SP263, 28-8 and 22C3 antibodies) showed that certain antibodies gave the same results for evaluation of the number of positive tumor cells on tissue sections, it would be interesting to perform a study comparing survival curves as a function of the expression of PD-L1 when evaluated with different clones. The study also showed a high level of cross over, in particular for patients treated with chemotherapy and then pembrolizumab, while taking into account the high level of toxicity of chemotherapy experienced by certain patients (2). Thus, for almost 50\% of the initially included patients a change in the choice of therapy was required.

Very recent results of clinical trials have also shown improved overall survival compared to chemotherapy for patients with advanced stage or metastatic NSCLC treated with pembrolizumab but with a much lower threshold of 
PD-L1 positivity than $50 \%$ positive tumor cells $(4,5)$. Thus, patients are eligible for pembrolizumab if more than $1 \%$ of tumor cells express PD-L1 (4,5). Another encouraging study showed that the association of pembrolizumab with chemotherapy gave a longer overall survival than patients treated with chemotherapy alone but this was independent of the percentage of PD-L1 IHC positivity on tumor cells (6).

PD-L1 IHC is still the only valid predictive biomarker for first-line immunotherapy of advanced stage or metastatic NSCLC (as long as the patient's tumor does not present a mutation in EGFR or a rearrangement in $A L K$ or ROS1 and/or the patient has untreated on brain metastases). While in a recent study this biomarker is not indispensable for administration of chemotherapy associated with pembrolizumab it should be evaluated for patients who cannot receive chemotherapy (fragile patients). Thus, patients showing positivity on at least $50 \%$ of tumor cells could receive an alternative treatment with immunotherapy alone. The limitations of PD-L1 IHC as a predictive biomarker have been extensively reported but could be further discussed with regard to a positive threshold of $1 \%$ of tumor cells $(7,8)$. The performance of the clones, even though globally similar for certain clones (SP263, 22C3, 28-8 in particular), does not seem to be identical for a low positive threshold. In particular, some studies show that negative labeling with the 22C 3 anti-PD-L1 can be positive with SP263 (9). The tumor heterogeneity is a major factor for consideration in the case of a $1 \%$ threshold, notably when evaluation is done on small-sized tissue samples, in particular bronchial biopsies, or even transthoracic biopsies. This tissue heterogeneity leads to differences in the level of expression of PD-L1 with different PD-L1 clones for biopsies and surgical specimens from the same patient $(10,11)$. The inter-observer variation is certainly more marked for a positive threshold of $1 \%$ than for a threshold of $50 \%$, so frequent external quality control must be performed $(7,12)$. Finally, the threshold of $1 \%$ may turn out to be more difficult to master with cytological samples, such as those of echo-guided transbronchial biopsies (13). Thus, indication of first-line immunotherapy based on a PD-L1 threshold of $1 \%$, as appears in several studies that have now been validated for daily practice, must certainly be considered in institutions associating specialist centers and experts in thoracic pathology.

While the use of PD-L1 IHC as a predictive biomarker has its limits with regard to the predictive value for some patients who may benefit from first-line immunotherapy, it is as yet the only approach authorized in daily practice to decide to treat or not treat patients. Despite the promising results as a predictive biomarker, the tumor mutation burden (TMB) also shows some limitations and to date has not been validated for routine clinical use (14). Even though it is a biomarker independent of PD-L1 to predict response, it has been shown recently that the TMB may show a number of limitations common with PD-L1 IHC, including heterogeneous expression, variable thresholds of positivity depending on the panel used and the therapeutic molecules considered as well as the need of studies harmonization and into the standardization of possible inter-platform variation (14-16). It is certain that the addition of the value of the TMB into the KEYNOTE-24 may provide supplementary information and identify other sub-groups of patients who respond or do not respond to pembrolizumab.

In conclusion, the KEYNOTE-24 update confirms the overall survival benefit of patients on pembrolizumab compared to standard treatment and opens the way to optimizing the initial published data. Finally, it seems that pembrolizumab improves overall survival across the PDL1 subgroups. This highlights the urgent need to identify more robust predictive biomarkers than PD-L1 for a better stratification of patients receiving immune check point inhibitors.

\section{Acknowledgments}

The author thanks the Cancéropôle PACA, the Ligue Départementale de Lutte contre le Cancer des Alpes Maritimes and the Conseil Départemental des Alpes Maritimes, for their support.

\section{Footnote}

Conflicts of Interest: P Hofman is a member of different industrial scientific advisory boards (Roche, AstraZeneca, Bristol-Myers Squibb, Pfizer, Novartis, Merck, MSD, Qiagen, Thermofischer, Biocartis) for which he receives honorarium

\section{References}

1. Reck M, Rodríguez-Abreu D, Robinson AG, Hui R, et al. KEYNOTE-024 Investigators. Pembrolizumab versus Chemotherapy for PD-L1-Positive Non-Small-Cell Lung Cancer. N Engl J Med 2016;375:1823-33.

2. Reck M, Rodríguez-Abreu D, Robinson AG, et al. Updated Analysis of KEYNOTE-024: Pembrolizumab 
Versus Platinum-Based Chemotherapy for Advanced NonSmall-Cell Lung Cancer With PD-L1 Tumor Proportion Score of 50\% or Greater. J Clin Oncol 2019;37:537-46.

3. Vigliar E, Malapelle U, Iaccarino A, et al. PD-L1 expression on routine samples of non-small cell lung cancer: results and critical issues from a 1-year experience of a centralised laboratory. J Clin Pathol 2019. [Epub ahead of print].

4. Expands pembrolizumab indication for first-line treatment of NSCLC (TPS $\geq 1 \%$ ). Accessed April 11, 2019. Available online: https://www.fda.gov/Drugs/InformationOnDrugs/ ApprovedDrugs/ucm635857.htm.FDA

5. Lopes G, Wu YL, Kudaba I, et al. Pembrolizumab (pembro) versus platinum-based chemotherapy (chemo) as first-line therapy for advanced/metastatic NSCLC with a PD-L1 tumor proportion score (TPS) $\geq 1 \%$ : Openlabel, phase 3 KEYNOTE-042 study. J Clin Oncol 2018;36:LBA4.

6. Gandhi L, Rodríguez-Abreu D, Gadgeel S, et al. KEYNOTE-189 Investigators. Pembrolizumab plus Chemotherapy in Metastatic Non-Small-Cell Lung Cancer. N Engl J Med 2018;378:2078-92.

7. Hofman P. PD-L1 immunohistochemistry for non-small cell lung carcinoma: which strategy should be adopted? Expert Rev Mol Diagn 2017;17:1097-108.

8. Lantuejoul S, Damotte D, Hofman V, et al. Programmed death ligand 1 immunohistochemistry in non-small cell lung carcinoma. J Thorac Dis 2019;11:S89-101.

9. Munari E, Rossi G, Zamboni G, et al. PD-L1 Assays 22C3 and SP263 are Not Interchangeable in Non-Small Cell Lung Cancer When Considering Clinically Relevant Cutoffs: An Interclone Evaluation by Differently Trained

Cite this article as: Hofman P. First-line immunotherapy for patients with advanced stage or metastatic non-small cell lung cancer... finally what threshold of PD-L1 expression on tumor cells? Transl Lung Cancer Res 2019;8(5):728-730. doi: $10.21037 /$ tlcr.2019.04.18
Pathologists. Am J Surg Pathol 2018;42:1384-89.

10. Elfving H, Mattsson JSM, Lindskog C, et al. Programmed Cell Death Ligand 1 Immunohistochemistry: A Concordance Study Between Surgical Specimen, Biopsy, and Tissue Microarray. Clin Lung Cancer 2019. [Epub ahead of print].

11. Ilie M, Long-Mira E, Bence C, et al. Comparative study of the PD-L1 status between surgically resected specimens and matched biopsies of NSCLC patients reveal major discordances: a potential issue for anti-PD-L1 therapeutic strategies. Ann Oncol 2016;27:147-53.

12. Ilie M, Hofman P. Reproducibility of PD-L1 assessment in non-small cell lung cancer-know your limits but never stop trying to exceed them. Transl Lung Cancer Res 2017;6:S51-4.

13. Ilie $\mathrm{M}$, Juco J, Huang L, et al. Use of the $22 \mathrm{C} 3$ antiprogrammed death-ligand 1 antibody to determine programmed death-ligand 1 expression in cytology samples obtained from non-small cell lung cancer patients. Cancer Cytopathol 2018;126:264-74.

14. Heeke S, Hofman P. Tumor mutational burden assessment as a predictive biomarker for immunotherapy in lung cancer patients: getting ready for prime-time or not? Transl Lung Cancer Res 2018;7:631-8.

15. Yarchoan M, Albacker LA, Hopkins AC, et al. PD-L1 expression and tumor mutational burden are independent biomarkers in most cancers. JCI Insight 2019;4. doi: 10.1172/jci.insight. 126908.

16. Zhang Y, Chang L, Yang Y, et al. The correlations of tumor mutational burden among single-region tissue, multi-region tissues and blood in non-small cell lung cancer. J Immunother Cancer 2019;7:98. 